\title{
Arm Motion Reconstruction via Feature Clustering in Joint Angle Space
}

\author{
Jack DiGiovanna, Student Member, IEEE, Justin C. Sanchez, Member, IEEE, B.J. Fregly, and Jose C. \\ Principe, Fellow, IEEE
}

\begin{abstract}
We hypothesize that a set of movemes can be used to reconstruct biomechanically realistic movements. Using parameters from a reaching and grasping task we create a representative three-dimensional motion. From this motion we extract features from the joint angle space. We believe that the physiological importance of these features makes them worth investigating as possible movemes. Machine learning techniques are employed to cluster similar features. The clusters are then used to recursively reconstruct the motion trajectory.
\end{abstract}

Even with only twenty clusters, the average trajectory reconstruction error in Cartesian space is less than $1 \%$ of the dynamic range of motion.

Our ability to create and analyze realistic motions may be crucial to both future BMI experiments where a desired signal is not available and our understanding of motor control.

\section{INTRODUCTION}

$\mathrm{T}$ HE development of motor control brain-machine interfaces (BMI) is faced with the challenge of finding a functional relationship between neuronal activity (collected from the cortex of the brain) and directed hand/arm movements [1]-[5]. A future goal of BMI design is use an engineered interface to restore mobility to paralyzed individuals [5]. Current BMI experimental paradigms utilize supervised learning techniques in conjunction with an inputoutput model (i.e. linear filter, neural network) to correlate recorded neural modulation with behavior generated by the patient [6].

In the ultimate embodiment of a BMI as used by a paralyzed patient, the training of the interface becomes a problem due to the lack of access to a desired (i.e. hand trajectory) signal [7]. Traditional supervised learning techniques may not be capable of finding the functional relationship between the patient's intent (neuronal

This work was supported in part by the National Science Foundation under Grant \#CNS-0540304, Children's Miracle Network, Tarr Charitable Family Foundation, and the Tillie, Jennie \& Harold Scwartz Foundation.

J. DiGiovanna is with the Department of Biomedical Engineering, University of Florida, 106 BME Building. Gainesville, FL 32611 USA (email: digiovaj@cnel.ufl.edu)

J. C. Sanchez is with the Department of Pediatrics, Division of Neurology, University of Florida, P.O. Box 100296, JHMHC, Gainesville, FL 32610 USA (e-mail: justin@cnel.ufl.edu)

B.J. Fregly is with the Department of Mechanical and Aerospace and Biomedical Engineering, 231 MAE-A Building, University of Florida, Gainesville, FL 32611 USA (e-mail: Fregly@ufl.edu)

J. C. Principe is with the Department of Electrical and Computer and Biomedical Engineering, NEB 451, University of Florida, Gainesville, FL 32611 USA (e-mail: principe@cnel.ufl.edu) modulation) and particular hand/arm trajectory due to the paradigm shift.

To contend with this problem, we are hypothesizing that a bottom-up approach can be used in the motor control scheme to artificially generate stereotyped but biomechanically realistic movement. The movement is deconstructed into elemental components - "movemes." Combinations of movemes may be useful as a desired signal for BMIs in cases where the patient cannot generate a real trajectory. The concept of using movemes (also referred to in the literature as motion primitives), or building blocks of movement, has been suggested by experts in motor control [8]-[13] where their presumed function is to reduce the dimensionality of the space where control solutions are sought. This type of "divide and conquer" approach has been applied in speech processing where phonemes have been shown to be a computationally efficient method to reconstruct speech [14]. In the case of BMIs, the first step is to define movemes and test how well they perform in trajectory reconstruction.

There is no consensus on how exactly to define a moveme. Our approach consists of two components: define a biologically plausible movement trajectory and then define similar features in the movement trajectory as a moveme. There have been a variety of prior approaches where researchers use different techniques to extract a piecewise representative feature from motion trajectory data. The motion data can include electromyographic (EMG) activations, joint angle trajectories, joint torques, endpoint trajectories, and relevant derivatives of those quantities [8][10].

A common goal in the researcher's approaches has been to show some biological plausibility in their results. Hwang and Shadmehr created muscle spindle models and utilize the firing of those models in a reaching task [11]. Todorov and Ghahramani created a dynamic arm model with two joints, four muscles, individual muscle spindles, and Golgi tendon organs with some simplifications [8]. Both approaches yielded some success in representing two-dimensional motion using only a subset of basis functions.

We propose to build on the work of prior researchers by extracting features from a biologically inspired threedimensional motion. The motion is created using end points and timing from a behavioral experiment and a simplified upper extremity skeletal model developed from healthy human subjects [15]. The motion is generated using inverse 
dynamics optimization [16] of a cost function found in biomechanical modeling [17], [18].

Features are extracted from the joint angle space for both biological and practical motivations. Muscle lengths and velocities are determined based on the angular positions and velocities of the joints that the muscle spans. There are known physiological relationships between muscle force generation and both muscle length and muscle velocity [19], [20]. Identical muscle activations (from neural signals) will create different muscle forces depending on these two relationships. The muscle's moment arm across a joint is determined by the ratio of change in muscle length to change in joint angle. The torque produced at a joint is the sum of muscle forces multiplied by their moment arms across that joint. We believe these three physiological relationships suggest that joint angle relationships are very important and may serve as a descriptor of different states of the arm. Although we do not use individual muscles in our model, we feel that these different arm states may correspond to different movemes.

The practical motivation for working in joint angle space is that we plan to use a set of linear filters with weights determined by the extracted features to reconstruct joint angle trajectories. If the features were extracted from the Cartesian space this would not be prudent due to the nonlinear transformation between that Cartesian and joint angle space. Errors can still be compared in Cartesian space because there is a unique mapping from joint angles to endpoints; however, the converse mapping is not unique.

Once the joint angle features are defined, machine learning techniques are employed to group similar features. These feature groups will be used for trajectory reconstruction; their relationship to movemes will be explored in the Discussion section.

\section{METHODS}

\section{A. Initial Trajectory Definition}

We consider reaching and grasping to be a critical movement task for restoring independence to paralyzed individuals. An example of such a task is moving one's hand from a resting position to a glass of water and picking it up.

We developed a synthetic reaching motion using an upper extremity computer model. A simplified version of the previously published human arm model [15] possessing five degrees of freedom (DOFs) was used for the investigation, with the shoulder possessing two DOFs, the elbow two DOFs, and the wrist one DOF. The model was created using experimentally derived joint kinematics and limb segment inertias [15]. The model was actuated by joint torques rather than individual muscles for two reasons. First, the set of five joint torques provides a lower dimensional workspace than the fifty muscles included in the original model. Second, to solve for muscle forces requires a further optimization at each time step in the motion, assigning muscle forces based on a hypothesized cost function.

Development of the synthetic reaching and grasping motion proceeded in two stages: a robotics-based initial definition and a biomechanically-based optimization. We defined an initial trajectory that satisfied known constraints. The constraints were chosen to match a reaching experimental paradigm, which we have previously used to record neural data from primary motor cortex (MI) in BMI experiments [21]. There are three constraints based on the hand location at rest, when it contacts the target, and when the target is moved. There are also two velocity constraints such that the initial and final angular velocities (and correspondingly endpoint velocities) will be zero. The position constraints were scaled to match the skeletal model [15], and the joint angles in the model were then adjusted manually until the arm reached the constraint locations in space. All of the joint angles at these positions were recorded. The initial trajectory was then defined using the given constraints by applying a cubic polynomial equation independently to each of the joints. The coefficients of the equations were determined based on the joint angles (initial, target contact, and final), joint angular velocities (initial, target contact, and final), and the total time for the motion. The equation for each joint is given in the appendix; this method is used because it is a "standard practice for reconstructing smooth trajectories." [22]. The movement time was set at $500 \mathrm{~ms}$ based on observed performance of multiple subjects in the experimental paradigm.

\section{B. Trajectory Optimization}

The initial motion trajectories were then optimized using a biomechanical modeling cost function. The cost $(J)$ function given in (1) minimizes the weighted sum of squared changes in joint torques $\left(\mathrm{d} T_{j} / d t\right)$ [17], [18]. In (1) $n j$ is the number of joints and $t f$ is the final time. Although nested optimization methods exist to determine the cost function weights that best match given motion data [23], those approaches are complex and computationally intensive. Therefore we used a common approach in the neural control and biomechanics literature [17], [18] and ignored the impact of the weights $\left(w_{j}\right)$ by setting them all equal to one.

$$
J=\frac{1}{n j} \sum_{j=1}^{n j} w_{j} \sum_{t=1}^{t f}\left(\frac{d T_{j}(t)}{d t}\right)^{2}
$$

A sequential quadratic programming algorithm (Matlab's fmincon function) was used in the optimization due to the presence of constraints in the problem formulation. The inverse dynamics equations are highly nonlinear and complex and must be re-derived in the event of any model changes. To solve these equations we used a generalpurpose musculoskeletal modeling system [24] to calculate the torques at each $1 \mathrm{~ms}$ step of the motion using inverse dynamics analysis. The constraints on this optimizer were set such that the joints could not exceed their physiological range and that the constraints in the initial trajectory 
definition would be satisfied. Each joint angle trajectory was parameterized using 10 node points fitted with cubic interpolating splines to provide position, velocity, and acceleration values at any desired time. These node points were used as design variables in the optimization. Fig. 1 shows the initial motion and optimized motion for shoulder flexion over the entire reach and grasp task. The optimized joint trajectories are not solved independently because the torque at any joint affects the motion of all other joints in the linkage via dynamic coupling [22]. This situation is more appropriate in modeling physiological control.

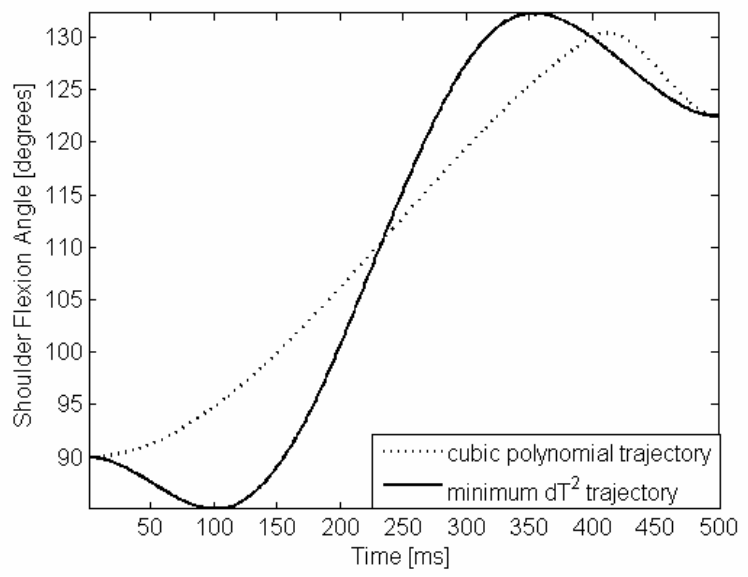

Fig. 1. Optimized shoulder flexion angle during a reach and grasp motion.

\section{Feature Definition}

We define a feature (and potentially movemes) by the relationship between the current set of joint angles to the next set of joint angles. These features $(v)$ are defined in (2) where $\theta$ is the joint angle, $j$ is the joint number, and $\Delta t$ is $1 \mathrm{~ms}$. These features provide direct information about joint angular velocity. Although joint angle position is not directly provided, it can be inferred based on initial conditions or a neural network could learn which moveme to switch to based on the prior and current $v$. For the $500 \mathrm{~ms}$ motion there are 499 five-dimensional feature vectors.

$v(t, j)=\theta_{j}(t+\Delta t)-\theta_{j}(t)$

\section{Feature Projection in Three DOF}

The features and cluster centers from the optimized motion were projected onto the three dimensions where the features exhibited the highest variance to visualize the clustering performance (see Fig. 2). The three axes represent shoulder flexion, elbow flexion, and wrist flexion. The result is a smooth trajectory of features throughout the motion. We projected the features using different joint combinations and in all cases, a smooth trajectory exists. However, this smooth trajectory is not equally distributed and can be segmented with standard clustering algorithms.

While these results are encouraging, it is premature to reach any conclusions about the relationship between our features and movemes. Movemes are yet to be defined, so it is unclear how many are included in the motion. If we draw comparisons to speech, the entire motion could be analogous to a single word. For example, there are only two phonemes in the words "show" or "high". It would be unreasonable to expect to extract a large set of phonemes from a single word. The counter-argument is that the entire motion should be compared to a sentence, in which case a larger set of phonemes could be extracted. Without a priori knowledge of the correct number of movemes we can only use clustering error as a guide to how many feature groups to use. In the Discussion section we propose a method for validation of feature clusters as movemes.

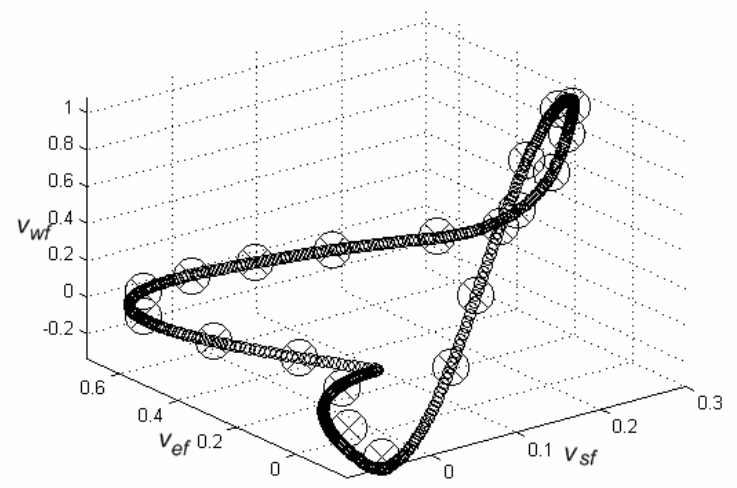

Fig. 2. All motion features projected onto 3 dimensions. Axes are shoulder flexion $\left(v_{s f}\right)$, elbow flexion $\left(v_{e f}\right)$ and wrist flexion $\left(v_{w f}\right)$. Small circles represent features; larger $\mathrm{X}$-filled circles represent cluster centers

\section{E. Feature Clustering}

The idea of movemes is to find a small subset of basis movements that can represent any motion. If all of the feature vectors were used, the motion could be perfectly reconstructed. To find a subset of features we can use traditional clustering techniques to partition the feature space to find some structure in the data. Two clustering approaches were used to try to find an optimal number of clusters centers and to compare the results for a range of cluster centers. The first approach allowed cluster dropping. The initial cluster centroids were uniformly distributed random numbers within the range of the feature vectors.

In the second clustering approach, the initial cluster centroids were randomly chosen from coefficient samples and cluster dropping was not allowed. In the event of an empty cluster, a new cluster centroid was created using the sample that is furthest from its current cluster label. Both clustering methods minimized the squared Euclidean distance between the cluster centers and their members (clustering error). K-means (Matlab's kmeans function) clustering was used for both approaches due to its computational efficiency and it performance with the feature vectors. 


\section{RESULTS}

\section{A. Feature Clustering Performance}

The cluster dropping approach was tested in 100 Monte Carlo simulations of different initial centroids to find an optimal number of clusters for the motion. The optimal number was found to be 42 clusters. The fixed cluster number approach was tested to find the clustering error for a range of sizes (2 to 75 clusters). For each cluster size, 100 Monte Carlo simulations of different initial centroids were performed to find the minimum clustering error. Fig. 3 shows the optimal clustering error vs. the number of clusters. There is an initial sharp reduction in clustering error as the number of clusters increases; however, beyond approximately 15 clusters there are diminishing returns.

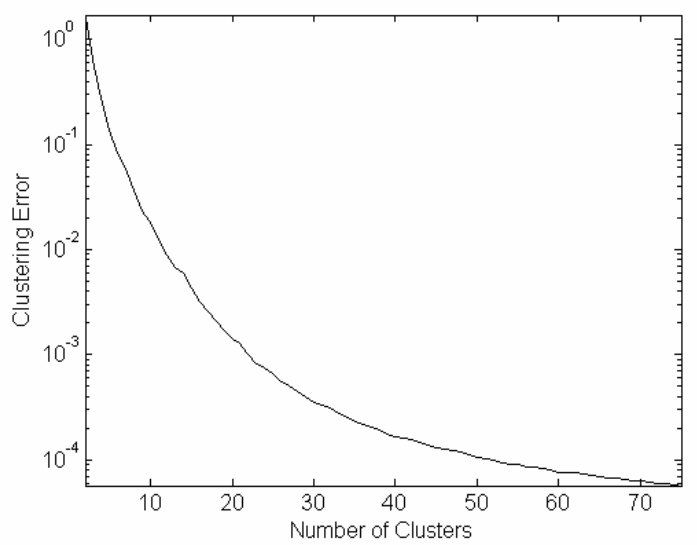

Fig. 3. Clustering error as a function of number of clusters.

\section{B. Trajectory Reconstruction}

The feature clusters are tested for their utility in reconstruction of the synthetic motion in both joint angle and Cartesian space. Each cluster is added to the initial true joint angle vector to create estimates of the next joint angle vector. These estimates are all compared with the next true joint angle vector to find which one creates the minimal error. That estimate is then fed back to the input (see Fig. 4). For all time greater than $1 \mathrm{~ms}$, the trajectory is reconstructed recursively.

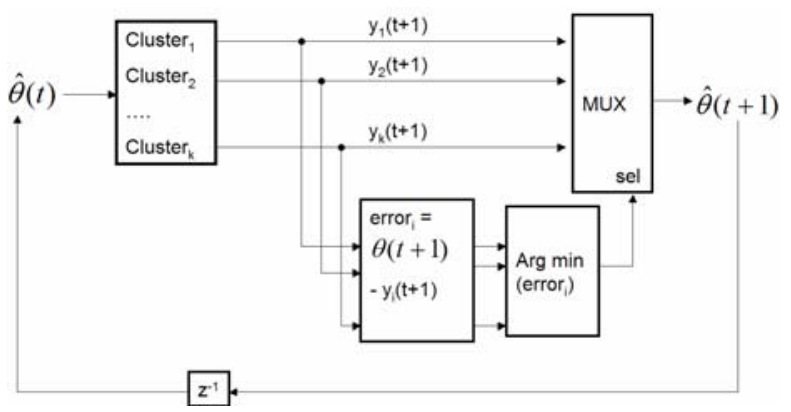

Fig. 4. Block diagram of trajectory reconstruction.

It is expected that any errors that occur in trajectory reconstruction should propagate through the motion. However, we will show in the RMS Joint Angle Errors section that this is not the case, the reconstruction can compensate for prior errors.

Fig. 5 shows the optimal RMS error in Cartesian coordinated vs. the number of feature clusters. (In this case the clusters are picked based on the minimal clustering error, though the associated Cartesian errors may not be minimal.) As in fig. 2, there is a sharp initial drop in error; then continuous improvement with increasing numbers of clusters.

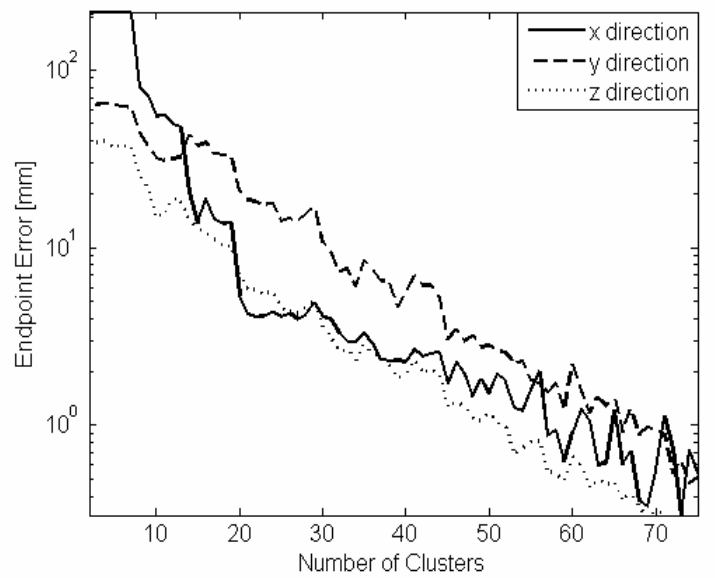

Fig. 5. RMS endpoint errors vs. number of clusters. This error is not normalized. If RMS errors are divided by dynamic range, they are $0.8 \%, 1.1 \%$, and $0.6 \%(\mathrm{x}, \mathrm{y}$, and $\mathrm{z})$ at 20 clusters

Fig. 6 shows a portion of the reconstructed trajectory of elbow flexion. This portion was selected because it represented the greatest error. Otherwise the difference would not be visibly discernable in a plot.

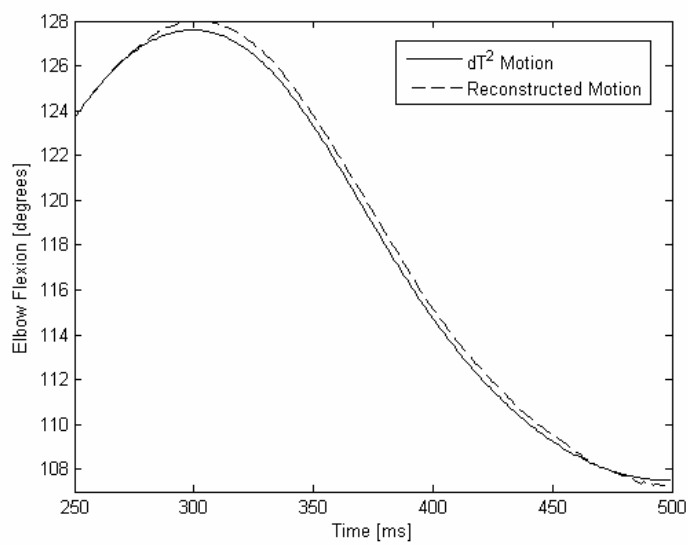

Fig. 6. Reconstruction of elbow flexion using 42 clusters. This portion of the motion exhibits the greatest discrepancy between the reconstructed and original motions.

When we investigated the Monte Carlo results, we noticed that there were groups of clusters that created lower reconstruction errors than the optimal (in terms of clustering error) group of clusters. These higher error clusters were not as representative of the original motion yet provided additional error-correction paths during reconstruction. However, this error reduction is related to the bias-variance dilemma in training adaptive filters. Although it is possible to include extra parameters so that bias is lower for the 
training motion, this practice may lead to higher variance in novel motions. Also, using clusters not firmly based in joint angle relationships violates our fundamental moveme assumptions and would confound the investigation.

\section{Cluster Selection}

The sequence of cluster selections in trajectory reconstruction is shown in fig. 7. There is no rigid timescale for the clusters to be active. This is consistent with the speech analogy where a phoneme only describes the position of the vocal articulators; the length a phoneme is expressed in time is arbitrary. There is also rapid switching between clusters for portions of the movement. These situations occur because there are not sharp divisions in feature space between the clusters (see fig. 3) and there is a slight error reduction achieved (see fig. 4). Imposing either a minimum error improvement or minimum distance between consecutive cluster centers before a switch occurs reduces, but does not eliminate, this rapid switching. However, this situation may not indicate a flaw with the feature clusters, but rather suggest that a blend of clusters is more appropriate than a winner-take-all approach for certain portions of the movement.

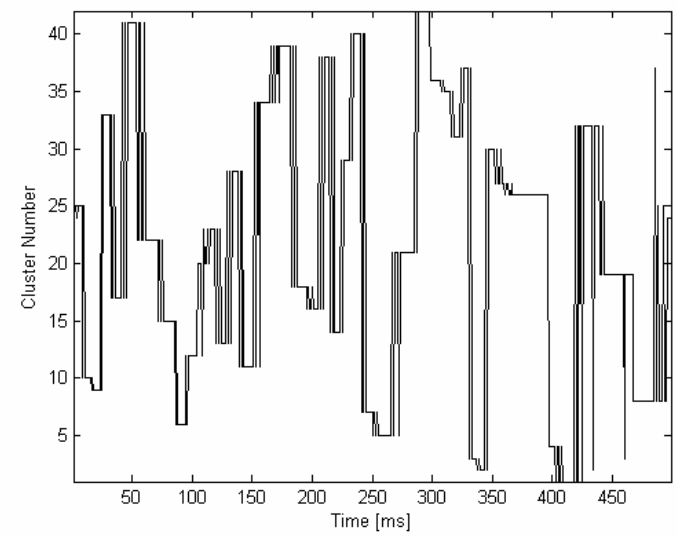

Fig. 7. Winning clusters throughout the motion. Like phonemes, these clusters are not rigidly defined in time. Cluster \#27 is active from $362 \mathrm{~ms}-400 \mathrm{~ms}$, yet cluster \#15 is only active from $80 \mathrm{~ms}-90 \mathrm{~ms}$.

\section{RMS Joint Angle Errors}

RMS joint angle errors between the reconstructed and optimized motion for elbow flexion and wrist flexion are shown in fig. 8. The dynamic ranges of the joints are 105 degrees and 100 degrees respectively. The errors were compared to the joint angular velocity and acceleration, but there were no obvious correlations between them. The effect of cluster switching can also be seen in fig. 8 (the cluster switch times are given in fig. 7) where the error seems jagged. The errors do improve for some joint angles throughout the motion. This improvement is promising because it shows that even with recursive motion reconstruction, the errors do not necessarily propagate through the entire motion.

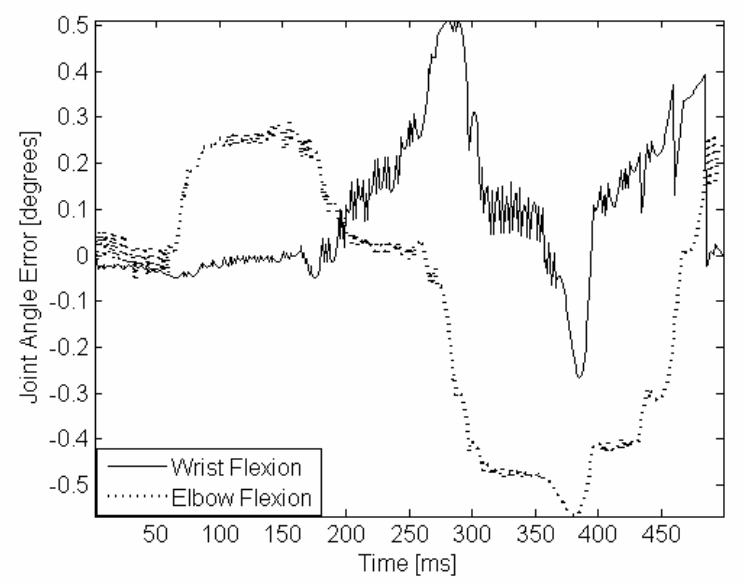

Fig. 8. RMS Joint angle errors. The rapid cluster switching times are seen in these errors (i.e. $420 \mathrm{~ms}-430 \mathrm{~ms}$ ).

\section{Discussion}

Here we developed an experimental architecture to segment a biologically plausible movement trajectory into its elemental components. The ability to specify such trajectories may provide an alternative desired signal for training BMIs for patients who are paralyzed. Machine learning techniques were used as a principled method to perform the segmentation. Based upon the techniques applied here, we able to recursively reconstruct the trajectory with high degree of accuracy. We found that there is an optimal set of movemes (this set is smaller than $10 \%$ of the total number of features) that best parameterized the particular movement space for producing the best reconstruction.

Moreover, the selection of movemes may not be a hard boundary as was initially hypothesized. This observation can be attributed either to machine learning assumptions provided by the errors in the clustering or by biological precepts that may effectively use combinations of movemes in practice. Answering which one is correct is the subject of future research. Nevertheless we have evaluated here a technique for clustering some features of the trajectory. We also tested this approach with a different biomechanical cost function optimization and that it

In order to validate that our cluster indeed represent movemes we need to use neural data. We hypothesize that neural networks can be used to select which local models are appropriate at the current time based on which moveme(s) are contributing to the motion. The coupling of developing movemes for arbitrary reaching trajectories with neural activity with hopefully provide an alternative solution for training BMI for paralyzed patients

\section{APPENDIX}

The cubic polynomial equation for computing the initial joint angle $(\theta)$ paths is given in (3) and the coefficients are defined in (4). The variable notation is 
consistent with the Trajectory Optimization subsection of Methods. This equation is given in [22].

$$
\begin{aligned}
& \theta(t)=a_{0}+a_{1} t+a_{2} t^{2}+a_{3} t^{3} \\
& a_{0}=\theta_{0} \\
& a_{1}=\dot{\theta}_{0} \\
& a_{2}=\frac{3}{t_{f}^{2}}\left(\theta_{f}-\theta_{0}\right)-\frac{2}{t_{f}} \dot{\theta}_{0}-\frac{1}{t_{f}} \dot{\theta}_{f} \\
& a_{3}=-\frac{2}{t_{t}^{3}}\left(\theta_{f}-\theta_{0}\right)+\frac{1}{t_{f}^{2}}\left(\dot{\theta}_{f}+\dot{\theta}_{0}\right)
\end{aligned}
$$

\section{REFERENCES}

[1] J. M. Carmena et al., "Learning to control a brain-machine interface for reaching and grasping by primates," PLoS Biology, vol. 1, pp. 116, 2003

[2] J. Wessberg et al., "Real-time prediction of hand trajectory by ensembles of cortical neurons in primates," Nature, vol. 408, pp. 361365, 2000

[3] A.B. Schwartz, D. M. Taylor, and S. I. H. Tillery, "Extraction algorithms for cortical control of arm prosthetics," Current Opinion in Neurobiology, vol. 11, pp. 701-708, 2001

[4] J.C. Sanchez, D. Erdogmus, Y. Rao, J. C. Principe, M. Nicolelis, and J. Wessberg, "Learning the contributions of the motor, premotor, and posterior parietal cortices for hand trajectory reconstruction in a brain machine interface," presented at IEEE EMBS Neural Eng. Conf, Capri, Italy, 2003

[5] J. P. Donoghue, "From mind to movement: developing neurotechnologies to restore lost function,” presented at Neural Information Processing Systems, Whistler, British Columbia, 2004

[6] J. C. Sanchez , S. P. Kim et al., "Input-output mapping performance of linear and nonlinear models for estimating hand trajectories from cortical neuronal firing patterns," presented at Int. Work on Neural Networks for Sig. Proc., Martigny, Switzerland, 2002

[7] G. J. Gage, K. A. Ludwig, K. J. Otto, E. L. Ionides, and D. R. Kipke, "Naïve coadaptive cortical control," J. Neural Eng., vol. 2, pp. 52-63, 2005.

[8] E. Todorov and Z. Ghahramani, "Unsupervised learning of sensorymotor primitives," in Int. Conf. IEEE EMBC 2003 Proc., New Jersey 2003, pp. 1750-1753.

[9] K. Thoroughman and R. Shadmehr, "Learning of action through adaptive combinations of motor primitives," Nature, vol. 407, pp. 742-747, October 2000.

[10] P. Morasso and F.A. Mussa-Ivaldi, "Trajectory formation and handwriting: A computational model,” Biol. Cybernetics, vol. 45, pp. 131-142, 1982.

[11] E.J. Hwang and R. Shadmehr, "Internal models of limb dynamics and the encoding of limb state,” J. Neural Eng., vol. 2, pp. s266-s278, August 2005.

[12] D.M. Wolpert and M. Kawato, "Multiple paired forward and inverse models for motor control,” Neural Nets, vol. 11, pp. 1317-1329, 1998.

[13] M. Kawato, "Internal models for motor control and trajectory planning,” Curr. Op. in Neurobiology, vol. 9, pp. 718-727, 1999.

[14] D. H. Klatt, "Structure of a phonological rule component for a synthesis-by-rule program,” IEEE Trans. Acoustic, Speech, and Sig. Proc., ASSP-24, pp. 391-398, October 1976.

[15] K. Holzbaur, W. Murray, and S. Delp, "A model of the upper extremity for simulating musculoskeletal surgery and analyzing neuromuscular control," Annals of Biomedical Eng, vol. 33, no. 6, pp. 829-840, June 2005.

[16] M. L. Nagurka and V. Yen, "Fourier-based optimal control of nonlinear dynamic systems," J. Dynamic Systems, Measurement, and Control, vol. 112, pp. 17-26, 1990.

[17] Y. Uno, M. Kawato, and R. Suzuki, "Formation and control of optimal trajectory in human arm movement - minimum torque-change model," Biological Cybernetics, vol. 61, pp. 89-101, 1989
[18] Y. Wada, Y. Kaneko, E. Nakano, R. Osu, and M Kawato, "Quantitative examination for multi joint arm trajectory planning using a robust calculation algorithm of the minimum commanded torque change model,” Neural Networks, vol. 14, pp. 381-393, 2001

[19] A. J. van den Bogert, K. G. M. Gerritsen, and G. K. Cole, "Human muscle modeling from a user's perspective.” J. Electromyography and Kinesiology,” vol. 8, pp. 119-124, 1998

[20] A.C. Guyton and J. E. Hall, Textbook of medical physiology, $10^{\text {th }}$ ed. Philadelphia, PA: W. B. Saunders Company, 2001, pp. $72-77$.

[21] J. C. Sanchez, J. C. Principe, P.R. Carney, "Is neuron discrimination necessary for linear and nonlinear brain machine interface models," accepted to $11^{\text {th }}$ Int. Conf. Human-Computer Interaction, 2005.

[22] J. Craig, Introduction to robotics: mechanics and control, $2^{\text {nd }}$ ed. Reading, MA: Addison-Wesley, 1989, pp. 230-236.

[23] C. Bottasso, A. Croce, S. Sartirana, and B. Prilutsky, "A method for inferring the optimization cost function of experimentally observed motor strategies," in Proc. ASME IDETC/CIE 2005, pp. 1-8.

[24] S. L. Delp, and J. P. Loan, "A graphics-based software system to develop and analyze models of musculoskeletal structures," Comput. Biol. Med, vol. 25, pp. 21-34, 1995. 